\title{
Pyoderma gangrenosum with monoclonal IgA gammopathy and pulmonary tuberculosis. Illustrative case and review
}

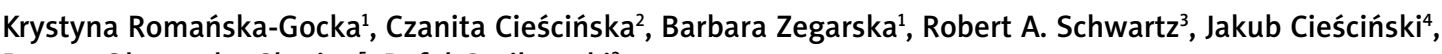 \\ Dorota Olszewska-Słonina ${ }^{5}$, Rafat Czajkowski²
}

\author{
1Department of Cosmetology and Esthetic Dermatology, Ludwik Rydygier Medical College in Bydgoszcz, Nicolaus Copernicus University \\ in Torun, Poland \\ Head of the Department: Prof. Barbara Zegarska MD, PhD \\ 2Department of Dermatology, Sexually Transmitted Diseases and Immunodermatology, Ludwik Rydygier Medical College in Bydgoszcz, \\ Nicolaus Copernicus University in Torun, Poland \\ Head of the Department: Rafat Czajkowski MD, PhD, DSc \\ ${ }^{3}$ Department of Dermatology and Pathology, Rutgers University New Jersey Medical School, Newark, New Jersey, USA \\ Head of the Department: Prof. Robert A. Schwartz \\ ${ }^{4}$ Department of Radiology and Diagnostic Imaging, Ludwik Rydygier Medical College in Bydgoszcz, Nicolaus Copernicus University \\ in Torun, Poland \\ Head of the Department: Zbigniew Serafin MD, PhD, DSc \\ ${ }^{5}$ Laboratory of Cell Biology and Genetics, Ludwik Rydygier Medical College in Bydgoszcz, Nicolaus Copernicus University in Torun, \\ Poland
}

Head of the Department: Dorota Olszewska-Stonina MD, PhD, DSc

Postep Derm Alergol 2015; XXXII, 2: 137-141 DOI: 10.5114/pdia.2014.40974

Pyoderma gangrenosum (PG) is a non-infectious inflammatory skin disease, resulting in large ulcers that can spread rapidly showing undermined, violaceous borders and a necrotic, purulent base [1-3]. The etiology remains unclear, but it appears to have an immunologic background [2-4]. It is probably a hyperergic reaction, connected with systemic disorders. Neutrophil dysfunction has been suggested (defects in chemotaxis or hyperreactivity). Evidence of abnormal neutrophil trafficking and metabolic oscillations has been described [4]. Circulating immunoglobulins affecting the neutrophil function and monoclonal or polyclonal hyperglobulinemia frequently occur in PG. Evidence suggests disturbances of immunoregulation; immunologic effector dysfunction occurs in some patients [5]. Furthermore, interleukin 8, a potent leukocyte chemotactic agent, is overexpressed in PG ulcers [6].

In up to $70 \%$ of cases, PG is associated with a variety of systemic diseases. The most common linkage is to inflammatory bowel disease, such as Crohn's disease and ulcerative colitis (30\%), rheumatoid or psoriatic arthritis (25\%), multiple myeloma, hematologic malignancy (particularly leukemia and monoclonal gammopathies), paraproteinemia (particularly lgA), viral hepatitis, diverticulitis, non hematological malignancy - prostate and renal adenocarcinoma, stomach carcinoid, and Kaposi's sarcoma $[4,7,8]$. There is also a rare familial form of PG, PAPA syndrome (pyogenic sterile arthritis, PG and acne), an autosomal dominant disorder resulting from a PSTPIP1 gene mutation [6]. Another syndrome with PG is PASH (PG, acne and suppurative hidradenitis) [4].

The distinctive clinical features of $P G$ are often sufficient to establish the diagnosis. However, diagnostic criteria have been articulated which require two major criteria and at least two minor criteria [4]. Major criteria: 1) Rapid progression of a painful, necrolytic, cutaneous ulcer, with an irregular, violaceous and undermined border, margin expansion of 1 to $2 \mathrm{~cm}$ per day or a $50 \%$ increase in ulcer size within 1 month. Pain is usually out of proportion to the size of the ulceration, typically preceded by a papule, pustule or bulla. 2) Other causes of cutaneous ulceration have been excluded - usually this necessitates skin biopsy or other investigations. Minor criteria: 1) History suggestive of pathergy or clinical finding of cribriform scarring. 2) Systemic diseases associated with PG. 3) Histopathologic findings (sterile dermal

Address for correspondence: Rafat Czajkowski MD, PhD, DSc, Department of Dermatology, Sexually Transmitted Diseases and Immunodermatology, Ludwik Rydygier Medical College in Bydgoszcz, Nicolaus Copernicus University in Torun, 9 Skłodowskiej-Curie St, 85-094 Bydgoszcz, Poland, phone: +48 5258545 68, e-mail: r.czajkowski@2gb.pl

Received: 29.12.2013, accepted: 13.01.2014. 
neutrophilia, mixed inflammation, lymphocytic vasculitis). 4) Treatment response (rapid response to systemic steroid treatment).

A 49-year-old woman was admitted to the Department of Dermatology, Sexually Transmitted Diseases and Immunodermatology, in November 2011, because of a rapidly expanding, necrolytic ulcer of the right leg. Three months earlier, the patient was diagnosed with pulmonary tuberculosis. After the Mantoux test, a painful nodule on an erythematous base appeared on the left forearm at the test site. This nodule progressed to an abscess and, after incision with drainage, healed with a thin scar (Figure 1). After a few days, another ulcer appeared suddenly on the formerly normal skin of the right leg and quickly expanded laterally to a diameter about $10 \mathrm{~cm}$ during a period of 2 weeks. It was painful, with violaceous, irregular, serpiginous undermined and well-defined borders, and developed a necrotic ulcerated center (Figure 2).

The diagnosis of pulmonary tuberculosis was confirmed by culturing Mycobacterium tuberculosis from the bronchoalveolar lavage fluid on Löwenstein-Jensen medium, and the patient was treated with tuberculostatics: rifampicin $450 \mathrm{mg}$ daily.

A biopsy specimen taken from the border of a leg ulcer showed a pseudoepitheliomatous hyperplasia of epidermis, massive neutrophilic infiltrate with suppurative inflammation, and necrotizing vasculitis, confirming the diagnosis of PG (Figure 3).

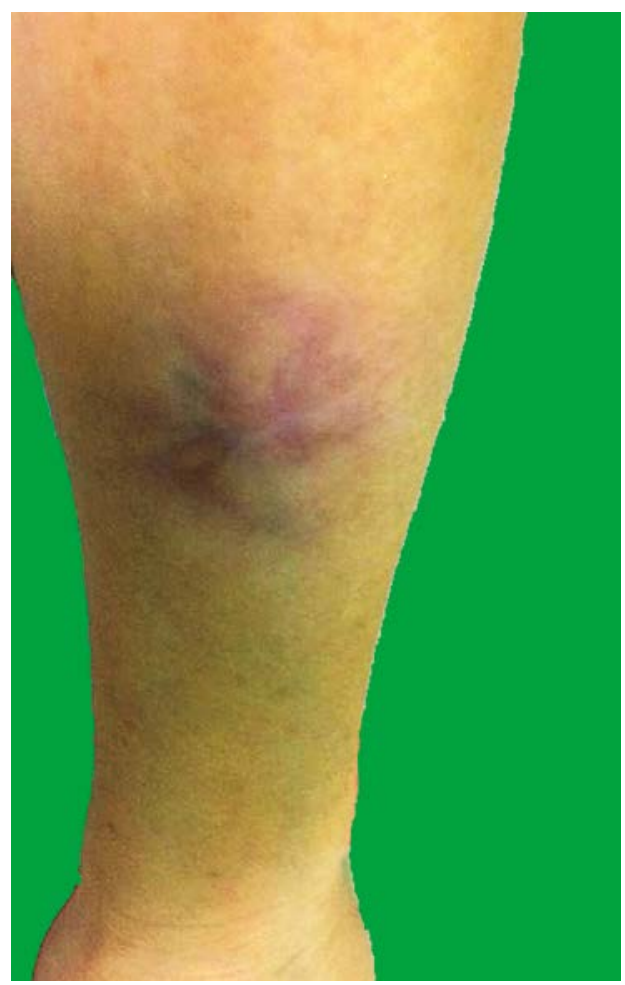

Figure 1. Scar on the forearm after healing the abscess in the site of Mantoux test (pathergy sign)
Laboratory tests revealed anemia, an elevated erythrocyte sedimentation rate, hypoalbuminemia, hyper $\beta 1$-globulinemia, hypogammaglobulinemia, and an elevated total immunoglobulin A (IgA) level of $18.60 \mathrm{~g} / \mathrm{l}$ (normal range: $0.70-4.00 \mathrm{~g} / \mathrm{l}$ ). The protein electrophoresis with immunofixation disclosed a monoclonal IgA $\kappa$-type gammopathy. Urine analysis did not demonstrate BenceJones protein.

Glucose, blood urea nitrogen (BUN), renal and liver function tests were within normal limits. Antinuclear antibodies (ANA) and anti-neutrophil cytoplasmic antibodies (ANCA) were negative. Bacterial swab and tissue culture were negative. Doppler ultrasonography of arteries and veins did not reveal any pathological changes.

The bone marrow trepanobiopsy showed an increased number of plasma cells. The patient was initially treated with prednisone (40 mg daily; $0.8 \mathrm{mg} / \mathrm{kg}$ b.w.) with rapid clinical improvement. After reducing the daily dose of prednisone to $20 \mathrm{mg}$, the ulcer recurred. Next treatment was prednisone (60 mg/day; $1.2 \mathrm{mg} / \mathrm{kg}$ b.w.) and dapsone $50 \mathrm{mg}$ daily was added as a steroid-sparing and non-suppressive agent. 0.1\% tacrolimus ointment was applied topically once daily. Significant improvement in the clinical status was noticed after 3 weeks of treatment when the ulcer diameter reduced to about $2 \mathrm{~cm}$, but one month later several new ulcerations appeared on the left leg. Simultaneously to reducing gradually the dose of prednisone, cyclosporine A was applied in dose

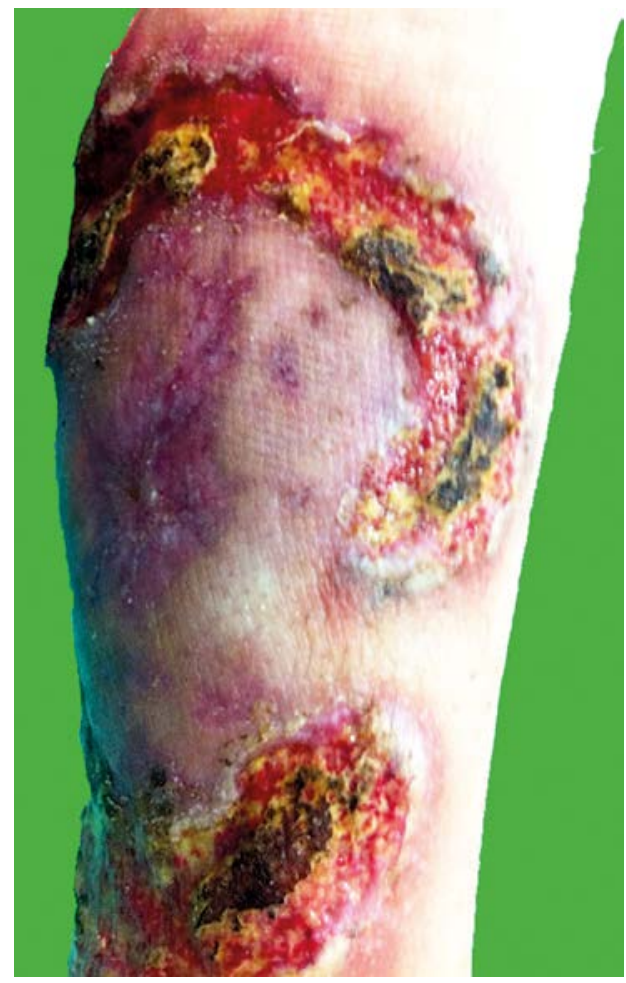

Figure 2. A large necrotic ulcer with typically violaceous, irregular, undermined borders on the leg 


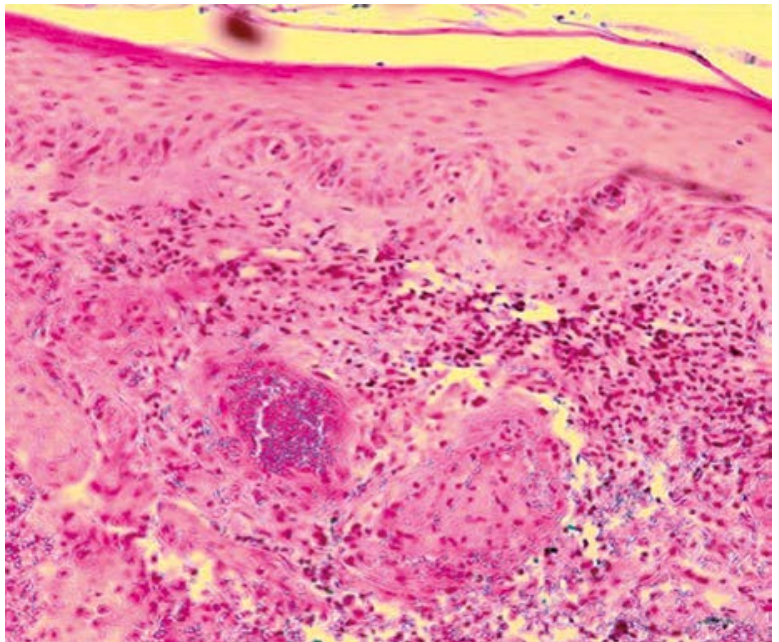

Figure 3. Histopathological biopsy from the undermined border revealed massive neutrophilic infiltrate (hematoxylin and eosin stain, 100x)

$5 \mathrm{mg} / \mathrm{kg}$ b.w. daily. The good results of treatment were documented after 1 month and complete healing with scarring was achieved after 6 months, without a relapse after cyclosporine A discontinuation (Figure 4).

Pyoderma gangrenosum is a persistent ulcerating disorder of unclear etiology often linked with systemic disease including ulcerative colitis and gammopathies, rarely Behcet's disease $[1,9,10]$. In 1967, Jablonska et al. reviewed earlier cases and reported 2 patients with PG and myeloma [2]. Pyoderma gangrenosum has usually a characteristic clinical appearance, as in the presented case. About $25-50 \%$ of patients give a history of skin trauma (pathergy sign) in the area of lesion development. In our patient, it was the site of the tuberculosis test where painful nodule appeared. The lesion develops a necrotic, ulcerated center, quickly expands laterally and it is exquisitely painful. The borders of the ulcer become erythematous to violaceous, irregular, and are classically well defined and undermined. As the patients is treated, the borders flatten and the ulcer heals with a thin, cribriform scar, typical for PG [6]. Interestingly, in our patient, the first lesion healed after surgical drainage of the abscess and the typical for PG clinical picture developed on the lower extremities, initially on the right leg, later on the left leg, without preceding trauma. The number of PG lesions can be different, ranging from one or two to multiple ulcers, sometimes in two separate anatomical locations. Pyoderma gangrenosum most frequently affects the lower extremities, as in the presented case, but can involve any cutaneous site [7, 11]. Possible areas of involvement described include the trunk, head and neck, groin, upper limbs, genitalia, abdomen, thighs, breast, and perianal region. Pyoderma gangrenosum ulcers are located on the lower extremities in about $75 \%$ of cases. When the ulcer is located elsewhere on the body, partic-

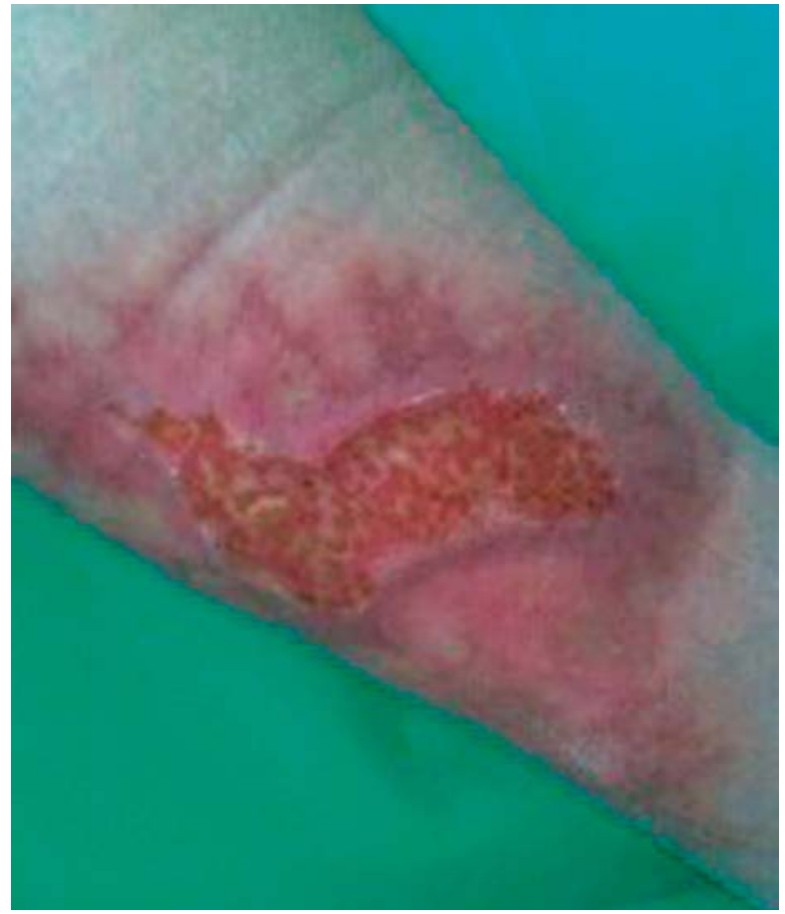

Figure 4. Healed pyoderma gangrenosum

ularly on the upper limbs and trunk, the clinician should suspect an etiology other than PG [12]. The histopathologic findings of PG are not specific; the primary objective in obtaining a biopsy specimen is to rule out other causes of ulceration. A biopsy taken from early lesions shows moderate perivascular infiltrate and endothelial swelling. Skin biopsy specimens, taken from the necrotic, undermined ulcer border of an active lesion, reveal mixed cellular inflammation with neutrophil predominance. A biopsy from an erythematous region adjacent to the ulcer may show vascular necrosis and thrombosis, erythrocyte extravasation and predominantly lymphocytic infiltrates [1, 2].

In the presented case we documented a significantly raised erythrocyte sedimentation rate and monoclonal IgA $\kappa$-type gammopathy. The association between monoclonal gammopathy of undetermined significance (MGUS) and PG is 7-9\% [3]. The MGUS is a premalignant plasma-cell proliferative disorder characterized by the presence of a monoclonal immunoglobulin and associated with a life-long average $1 \%$ annual risk of developing lymphoproliferative malignancies [13]. The prevalence of MGUS is about 3\% in the general population aged 50 years of age or older. Currently, there is no therapy to prevent this progression, so the standard treatment is observation alone [14, 15].

The differential diagnosis for leg ulcerations is vast and includes all causes of ulcerations with vascular etiologies being the most common such as vascular occlusive and venous diseases and vasculitis (atherosclerosis, 
vascular occlusion, atheroemboli) [12]. Other notable categories include cutaneous malignancies (squamous cell carcinoma, basal cell carcinoma, lymphomas, Kaposi's sarcoma, angiosarcoma), inflammation (lupus erythematosus, polyarteritis nodosa, Wegener's granulomatosis, panniculitis), primary cutaneous infections, metabolic disorders (diabetes mellitus), hematologic disorders (cryoglobulinemia), drug-induced ulcers (hydroxyurea, iodides, bromides, warfarin) or trauma (pressure, injury, burns) [16]. Infectious causes of ulcerative and pustular lesions that mimic PG include folliculitis, furunculosis, true pyoderma, ecthyma gangrenosum, superficial and subcutaneous mycoses, deep fungal infections such as histoplasmosis, cutaneous mycobacteriosis, syphilis, leishmaniasis, cutaneous viral infections and other cutaneous parasitic infections. Extracutaneous Crohn's disease may also be considered [12, 17].

Our patient fulfilled all of PG criteria described above: typical necrolytic cutaneous ulcer, confirmed as PG after histopathological biopsy, history of trauma, cribriform scar, associated disorder and quick response to corticosteroid treatment [4].

Multiple systemic and topical therapies have been described as being successfully in PG. This dermatosis generally responds quite well to multiple treatment regiments. Sometimes control of the underlying disease may lead to the resolution of PG. However, the activity of PG can be independent of the activity of the underlying disease so treatment of PG itself is still very important. Immunosuppressive management is the first line therapy unless there are contraindications [18]. Oral prednisone is the most common used medication at a dose of 1$2 \mathrm{mg} / \mathrm{kg}$ b.w. daily, together with topical application of corticosteroids such as clobetasol or topical immunomodulators such as tacrolimus or pimecrolimus, directly to the ulcer [19]. Pyoderma gangrenosum typically responds rapidly to moderate doses of systemic corticosteroids. Classically within $24-72 \mathrm{~h}$ after initiation of treatment the pain is rapidly alleviated. The most important clinical sign of therapeutic efficacy is a decrease in pain sensation [20].

In cases resistant to systemic corticosteroids, an alternative therapy should be given as well as if the ulcerations recur after corticosteroids are withdrawn. Consider increasing the immunosuppressive agent being used such as prednisone to $2 \mathrm{mg} / \mathrm{kg}$ b.w. daily or adding a second immunosuppressive agent [21].

Cyclosporine A had proved to be beneficial for patients resistant to corticosteroid therapy or who have had serious side-effects with it. Response of severe PG treated with cyclosporine A alone was seen within 1-3 weeks following administration at doses of $4.5-6.0 \mathrm{mg} / \mathrm{kg}$ b.w. daily, which also resulted in low relapse rates [18, 22, 23]. Some patients require low-dose maintenance therapy, but in others this drug can be completely withdrawn [2]. Although the side effects of cyclosporine A are known to be dose-dependent (nephrotoxicity, hypertension), patients on low-dose therapy should undergo periodic clinical and laboratory investigations [10, 23, 24].

Dapsone, sulfapiridine, and sulfasalazine are useful, but not all patients respond with the same satisfying treatment result. In the authors' experience, sulfasalazine is the most effective of the three above-mentioned medications. Initial daily doses range from 4 to $6 \mathrm{~g}$ and are gradually reduced to maintenance levels of $0.5-1 \mathrm{~g}$. Combining this with systemic corticosteroids in the initial phase of therapy may be necessary, but there have also been patients who failed to respond. Up to $200 \mathrm{mg} /$ day of dapsone has been used as a steroid-sparing agent [2, 25].

Infliximab is increasingly employed for PG in association with inflammatory bowel disease; there is accumulating evidence of its benefit in idiopathic PG, too [26]. Recent reports have indicated that recalcitrant PG can be effectively treated with immunomodulating agents with activity against tumor necrosis factor $\alpha$ (TNF- $\alpha)[27,28]$. Infliximab may be effective in patients with PG refractory to systemic corticosteroids and cyclosporine therapy. The onset of clinical response is fast and improvement often spectacular. In some cases, three pulses (0, 2 and 6 weeks) infused at $5 \mathrm{mg} / \mathrm{kg}$ were sufficient. To maintain remission, azathioprine can be administered. Appropriate caution is important in the selection and monitoring of patients treated with infliximab. All patients should be screened for latent tuberculosis [29].

An important disadvantage of treatment with azathioprine is the delayed onset of treatment effect, sometimes 8-12 weeks after initiation of therapy. Doses of 100-150 mg/day together with methylprednisolone $100 \mathrm{mg} /$ day, which could be reduced carefully when azathioprine became effective [6].

In the case of cyclophosphamide, recommended doses for severe PG are 1000 mg/week intravenously for the first 3 weeks, followed by $1-2 \mathrm{mg} / \mathrm{kg}$ b.w. orally in combination with low-dose corticosteroids [21]. The risk of infertility, hemorrhagic cystitis, hair loss and secondary malignancies must be taken into account [2].

Other methods of treatment reported to be successful include etanercept, mycophenolate mofetil, methotrexate, minocycline, mercaptopurine, thalidomide, clofazimine, and immunoglobulins [3, 30-32].

Another possible topical treatment includes dressings applied to the ulcers, usually normal saline-soaked gauze, silver impregnated, antiseptic and hydrocolloid dressing. Active topical treatment has a little impact on healing in severe cases but it avoids secondary infections. Topical therapy is usually ineffective in monotherapy but it can support systemic treatment. Surgical intervention should be avoided [33].

Despite advances in therapy, the long-term outcome for patients with PG remains unpredictable. Pyoderma gangrenosum is a potentially lethal disease. Poor prognostic indicators are male sex, old age at onset, and bul- 
lous PG, specifically when associated with malignant hematological disorders [34]. Even when patients respond well to therapy, relapses can occur in up to $70 \%$ of cases. Patients have a chance of healing the ulcers when treated rapidly and adequately, but in severe cases amputation of the involved extremity is also possible [20]. If the patient is not responding to treatment, re-evaluation to verify the diagnosis is warranted.

\section{Conflict of interest}

The authors declare no conflict of interest.

\section{References}

1. Birnkrant MJ, Papadopoulos AJ, Schwartz RA, et al. Pyoderma gangrenosum, acne conglobata, and IgA gammopathy. Int J Dermatol 2003; 42: 213-6.

2. Jablonska S. Zur Pathogenese des pyoderma gangrenosum. Hautarzt 1964; 15: 584-91.

3. Jablonska S, Stachow A, Dabrowska H. Rapports entre la pyodermite gangreneuse et le myelome. Ann Dermatol Syphilol (Paris) 1967; 94: 121-32.

4. Su WP, Davis MD, Weenig RH, et al. Pyoderma gangrenosum: clinicopathologic correlation and proposed diagnostic criteria. Int J Dermatol 2004; 43: 790-800.

5. Bergler-Czop B, Brzezińska-Wcisło L. Pyoderma gangrenosum in a patient with common variable primary immunodeficiency. Postep Derm Alergol 2013; 30: 188-91.

6. Ruocco E, Sangiuliano S, Gravina AG, et al. Pyoderma gangrenosum: an updated review. JEADV 2009; 23: 1008-17.

7. Binus AM, Quershi AA, Li VW. Pyoderma gangrenosum: a retrospective review of patients characteristics, comorbidities and therapy in 103 patients. Br J Dermatol 2011; 165: 1244-50

8. Wolska K, Michalska-Jakubus M, Pucuła J, et al. Bullous pyoderma gangrenosum associated with pancytopenia of unknown origin. Postep Derm Alergol 2014; 31: 272-6.

9. Conrad C, Trued RM. Pyoderma gangrenosum. J Dtsch Dermatol Ges 2005; 3: 334-42.

10. Langan SM, Powell FC. Vegetative pyoderma gangrenosum: a report of two new cases and a review of the literature. Int J Dermatol 2005; 44: 623-9.

11. Braun-Falco M, Kovnerystyy $\mathrm{O}$, Lohse $\mathrm{P}$, et al. Pyoderma gangrenosum, acne and suppurative hidradenitis (PASH) a new autoinflammatory syndrome distict from PAPA syndrome. J Am Acad Dermatol 2011; 66: 409-15.

12. Crowson AN, Mihm MC Jr, Magro C. Pyoderma gangrenosum: a review. J Cutan Pathol 2003; 30: 97-107.

13. Mansur AT, Balaban D, Goktay F. Pyoderma gangrenosum on the breast: a case presentation and review of the published work. J Dermatol 2010; 37: 107-10.

14. Saracino A, Kelly R, Liew D. Pyoderma gangrenosum requiring inpatient management: a report of 26 cases with follow up. Australas I Dermatol 2011; 52: 218-21.

15. Nguyen KH, Miller JJ, Helm KF. Case report and review of the literature an ulcers mimicking pyoderma gangrenosum. Int J Dermatol 2003: 42: 84-94.

16. Rongioletti F, Patterson JW, Rebora A. The histological and pathogenetic spectrum of cutaneous disease in monoclonal gammopathies. J Cutan Pathol 2008; 35: 705-21.

17. Stelmach-Gołdyś A, Czarkowska-Pączek B. Monoclonal gammopathy of underetmined significance - potential risk factor of mieloma multiple. Przegl Lek 2012; 69: 194-6.
18. Krasowska D, Kozłowicz K, Legieć W. Extensive leg and foot ulcers as a manifestation of cryoglobulinemia with accompanying monoclonal gammopathy of undetermined significance. Postep Derm Alergol 2011; 2: 145-9.

19. Dabade TS, Davis MD. Diagnosis and treatment of the neutrophilic dermatoses (pyoderma gangrenosum, Sweets syndrome). Dermatol Ther 2011; 24: 273-84.

20. Ricketts JR, Rothe MJ, Grand-Kels JM. Cutaneous stimulants of infectious diseases. Int J Dermatol 2011; 50: 1043-57.

21. Fatahzadeh M, Schwartz RA, Kapila R, et al. Orofacial Crohn's disease: an oral enigma. Acta Dermatovenerol Croat 2009; 17: 289-300.

22. Miller J, Yentzer B, Clark A. Pyoderma gangrenosum: a review and update on new therapies. J Am Acad Dermatol 2010; 62: 646-54.

23. Grubska-Suchanek E, Roszkiewicz J, Lange M. Pyoderma gangrenosum - result of cyclosporin A (Sandimmun Neoral) treatment. Przegl Dermatol 1999; 86: 263-8.

24. Khurrum Baig M, Marquez H, Nogueras JJ. Topical tacrolimus (FK506) in the treatment of recalcitrant parastomal pyoderma gangrenosum associated with Crohn's disease: report of two cases. Colorectal Dis 2004; 6: 250-3.

25. Ehling A, Karrer S, Klebl F. Therapeutic management of pyoderma gangrenosum. Arthritis Rheum 2004; 50: 3076-84.

26. Eichhorn PJ. Pyoderma gangrenosum. Dermatol Ther 2001; 14: 102-10.

27. Schofer H, Baur S. Successful treatment of postoperative pyoderma gangrenosum with cyclosporine. JEADV 2002; 16: 148-51.

28. Regueiro M, Valentine J, Plevy S. Infliximab for treatment of pyoderma gangrenosum associated with inflammatory, bowel disease. Am J Gastroenterol 2003; 98: 1821-6.

29. Patrone P, Bragadin G, De Francesco V. Pyoderma gangresnosum of the scalp treated with cyclosporine A. Int J Dermatol 2002; 41: 916-8.

30. Hewitt D, Tait C. Use of infliximab in pyoderma gangrenosum. Australas J Dermatol 2007; 48: 95-8.

31. Baglieri F, Scuderi G. Infliximab for treatment of resistant pyoderma gangrenosum associated with ulcerative colitis and psoriasis. A case report. Dermatol Ther 2010; 23: 541-3.

32. Brooklyn TN, Dunnill MG, Shetty A. Infliximab for the treatment of pyoderma gangrenosum: a randomized, double blind, placebo controlled trial. Gut 2006; 55: 506-9.

33. Charles CA, Leon A, Banta MR. Etanercept for the treatment of refractory pyoderma gangrenosum: a brief series. Int J Dermatol 2007; 46: 1095-9.

34. Laudanska H, Reduta T, Chodynicka B, Zdrodowska-Stefanow B. Pyoderma gangrenosum: report of two cases. Przegl Dermatol 1997; 84: 77-81. 